\title{
Identification and Characterization of Three Monilinia Species from Plum in China
}

Liang-Fen Yin, College of Plant Science \& Technology and the Experimental Teaching Center of Crop Science; Shu-Ning Chen, College of Plant Science \& Technology and the Key Lab of Crop Disease Monitoring \& Safety Control in Hubei Province, Huazhong Agricultural University, Wuhan 430070, China; Guo-Kang Chen, College of Plant Protection, Southwest University, Chongqing, 400715, China; Guido Schnabel, School of Agricultural, Forest and Environmental Sciences, Clemson University, Clemson, SC 29634; and Sheng-Fang Du, Chu Chen, Guo-Qing Li, and Chao-Xi Luo, College of Plant Science \& Technology and the Key Lab of Crop Disease Monitoring \& Safety Control in Hubei Province, Huazhong Agricultural University

\begin{abstract}
Yin, L.-F., Chen, S.-N., Chen, G.-K., Schnabel, G., Du, S.-F., Chen, C., Li, G.-Q., and Luo, C.-X. 2015. Identification and characterization of three Monilinia species from plum in China. Plant Dis. 99:1775-1783.

In total, 112 Monilinia spp. single-spore isolates were collected from plum fruit (Prunus salicina) symptomatic for brown rot disease from Yunnan, Hubei, and Zhejiang provinces and Chongqing municipality, China between 2012 and 2014. Three distinct colony morphologies (phenotypes) were observed on potato dextrose agar and two isolates per phenotype were selected for further analysis. Colony color, colony shape, conidia size, number of germ tubes per conidia, and pathogenicity on plum were investigated. The ribosomal internal transcribed spacer regions 1 and 2 as well as a polymerase chain reaction-based method that

amplified fragments of the glyceraldehyde-3-phosphate dehydrogenase $(G 3 P D H)$ and $\beta$-tubulin (TUB2) genes were used to identify the isolates to the species level. The three phenotypes were identified to be three different species: Monilinia fructicola, Monilia mumecola, and Monilia yunnanensis. Phylogenetic analysis based on G3PDH and TUB2 nucleotide sequences revealed that isolates within species clustered together regardless of host or geographical origin, suggesting that these factors did not play an important role for the evolutionary separation of the described species.
\end{abstract}

Plum (Prunus salicina) is a deciduous tree native to China and has more than 3,000 years of cultivation history. It is currently being planted in more than 20 provinces in China, and the production reached 5.6 million tons in 2010, representing approximately $57.5 \%$ of the world production (FAOSTAT, http://faostat.fao.org/site/339/ default.aspx). In China, plum is mostly sold as fresh fruit but dried fruit are also popular. The earliest reports of brown rot on stone fruit in China were made in the 1920s (Xiang 1957). Most studies on brown rot diseases in China focused on peach and only a few focused on brown rot of other stone fruit (Hu et al. 2011a,b; Yin et al. 2013; Zhu et al. 2005). The disease occurs every year and causes blossom blight, shoot dieback, twig cankers, and fruit rot. Outbreaks of the disease frequently occur and are severe in many instances. For example, in 2012 and 2013, the brown rot disease of plum severely affected the crop in production areas in the southwest of China, including Yunnan province and Chongqing municipality, according to a poll conducted among plum producers (C.-X. Luo, personal communication).

Despite the importance of the disease, there has been no recent survey to determine which Monilinia spp. are the causal agent in China. Until now, Monilia polystroma was the only species reported to cause brown rot on plum in China (Zhu and Guo 2010). Some species, including Monilinia fructicola and M. laxa, were found on fruit imported from Chile (Tian et al. 2008; Zhang et al. 2008). The goal of this study was to identify and characterize Monilinia spp. affecting plum in three different provinces and one municipality of China. Specific objectives were to (i) describe phenotypic variations among isolates, (ii) verify pathogenicity on detached plum fruit, (iii) identify isolates of different phenotypes to the species level, and (iv) infer phylogenetic relationships among isolates of different hosts and origins.

Corresponding authors: C.-X. Luo; E-mail; cxluo@mail.hzau.edu.cn; or G.-Q. Li; E-mail: guoqingli@mail.hzau.edu.cn

Accepted for publication 25 March 2015.

http://dx.doi.org/10.1094/PDIS-12-14-1308-RE

(C) 2015 The American Phytopathological Society

\section{Materials and Methods}

Nomenclature of Monilinia spp. In this study, the anamorph Monilia or teleomorph Monilinia were used to accurately refer to the specific organism that was found to cause brown rot disease. When we refer to just "Monilinia spp.", we are referring to all species.

Collection of Monilinia isolates from plum. Plum fruit with brown rot symptoms and signs were collected from the canopy of trees in Yunnan, Hubei, and Zhejiang provinces as well as Chongqing municipality during 2012 to 2014 . Conidia on diseased fruit were collected with sterile cotton swabs, and single-spore isolates were obtained as follows. Conidia were sprinkled on potato dextrose agar (PDA; $200 \mathrm{~g}$ of potato, $20 \mathrm{~g}$ of dextrose, and agar at $20 \mathrm{~g} \mathrm{liter}^{-1}$ ) by tabbing the swab over the petri dishes. Single spores were picked up with a glass needle under a converted COIC XSZ-4G compound light microscope (Chongqing Optical \& Electrical Instrument Co., Ltd., Chongqing, China) and transferred to new dishes with PDA. Individual colonies were transferred to PDA slants and incubated at $22^{\circ} \mathrm{C}$ in darkness for a week, then stored at $4{ }^{\circ} \mathrm{C}$ for further usage. In general, only one isolate was obtained from each fruit. In total, 30, 19, 33, and 30 isolates were collected from Yunnan, Hubei, Zhejiang, and Chongqing, respectively. Two $M$. polystroma reference isolates (CBS86 and CBS87) were obtained from the Centraalbureau voor Schimmelcultures, Utrecht, The Netherlands.

Colony morphology. Colony morphology was investigated on PDA medium. Plugs (6 $\mathrm{mm}$ in diameter) with actively growing mycelium were cut from the periphery of a 4-day-old colony and placed at the center of petri dishes $(9 \mathrm{~cm}$ in diameter) with fresh PDA, then incubated at $22^{\circ} \mathrm{C}$ in darkness. Based on distinct morphologies, two representative isolates of each morphological type were selected for further biological characterization (e.g., mycelial growth rate, stroma production, conidia size and germination style, and pathogenicity test). In order to investigate mycelia growth rate of different isolates, the diameter of colonies was measured 2, 3, 4, and 5 days after inoculation. Mean growth rates were calculated for each of the three replicates per isolate. Data were evaluated by one-way analysis of variance with a least significant difference test in SPSS Software (release 19.0; SPSS Inc., Chicago). Production of stromata for three replicates per isolate was investigated after 4 weeks of incubation at $22^{\circ} \mathrm{C}$ in darkness. 
Pathogenicity test. Pathogenicity of representative isolates with typical morphological characteristics was tested on detached plum fruit. Commercially mature 'Heibuling' plum were washed with tap water, then surface sterilized with $75 \%$ ethanol and rinsed with sterile water. A section of each fruit (6 $\mathrm{mm}$ in diameter) was removed with a cork borer and replaced with an agar plug extracted from the margin of a 4-day-old, actively growing culture on PDA. Fruit inoculated with PDA plugs without fungi were used as controls. The inoculated fruit were placed on small bottle caps in a plastic container with wet paper towels at the bottom and covered with a transparent plastic lid to maintain near $100 \%$ humidity. Fruit in containers were incubated at $22^{\circ} \mathrm{C}$ with $12 \mathrm{~h}$ of light and $12 \mathrm{~h}$ of darkness. The plastic lid was replaced with twice-folded gauze for aeration 3 days after inoculation. Three fruit were used for each isolate and the experiments were repeated. Conidia size, shape and germination styles were then investigated under a compound light microscope (Motic China Group Co., Ltd., Wuhan, China).

Conidia morphology. Conidia were harvested from inoculated plum fruit, the length and width of conidia were measured with a stage micrometer using a Motic BA200 light microscope (Motic

Table 1. Monilinia spp. isolates used for phylogenetic analysis

\begin{tabular}{|c|c|c|c|}
\hline \multirow[b]{2}{*}{ Host, isolate name } & \multirow[b]{2}{*}{ Species } & \multicolumn{2}{|c|}{ Origin $^{z}$} \\
\hline & & City, province & Country \\
\hline \multicolumn{4}{|l|}{ Plum } \\
\hline CCM13-1-2 & Monilinia fructicola & Hechuan, Chongqing & China \\
\hline CCM13-18-2 & M. fructicola & Hechuan, Chongqing & China \\
\hline CCM13-1-3 & M. fructicola & Yongchuan, Chongqing & China \\
\hline CCM13-5-1 & M. fructicola & Yongchuan, Chongqin & China \\
\hline CCM13-7-2 & M. fructicola & Yongchuan, Chongqing & China \\
\hline CCM13-3-1 & M. fructicola & Fuling, Chongqing & China \\
\hline CCM13-6-1 & M. fructicola & Fuling, Chongqing & China \\
\hline MCM13-14-1 & Monilia mumecola & Hechuan, Chongqing & China \\
\hline MCM13-6-1 & M. mumecola & Youyang, Chongqing & China \\
\hline MCM13-9-1 & M. mumecola & Youyang, Chongqing & China \\
\hline MCM13-12-1 & M. mumecola & Banan, Chongqing & China \\
\hline YYM12-57-3 & Monilia yunnanensis & Qujing, Yunnan & China \\
\hline YYM12-57-4 & M. yunnanensis & Qujing, Yunnan & China \\
\hline \multicolumn{4}{|l|}{ Peach, nectarine } \\
\hline Dmap3-08 & M. fructicola & Spartanburg, SC & United States \\
\hline Bmpc5 & M. fructicola & Byron, GA & United States \\
\hline ZM09-2a & M. fructicola & Hangzhou, Zhejiang & China \\
\hline HWL10-1b & M. mumecola & Wuhan, Hubei & China \\
\hline HXL10-4a & M. mumecola & Xiangfan, Hubei & China \\
\hline YKG10-61a & M. yunnanensis & Kunming, Yunnan & China \\
\hline SBG10-3a & M. yunnanensis & Baoji, Shanxi & China \\
\hline BSZGY-SZ-1 & Monilinia laxa & NA & Hungary \\
\hline Mfg4-GY-A & Monilia fructigena & NA & Hungary \\
\hline CBS- 86 & M. polystroma & NA & Japan \\
\hline CBS-87 & M. polystroma & NA & Japan \\
\hline \multicolumn{4}{|l|}{ Apricot } \\
\hline MHT13-1-1b & M. muтecola & Wuhan, Hubei & China \\
\hline MHT13-1-2b & M. mumecola & Wuhan, Hubei & China \\
\hline MHT13-1-3b & M. mumecola & Wuhan, Hubei & China \\
\hline MHT13-1-4c & M. mumecola & Wuhan, Hubei & China \\
\hline MHT13-2-2b & M. mumecola & Wuhan, Hubei & China \\
\hline MHT13-2-3b & M. mumecola & Wuhan, Hubei & China \\
\hline \multicolumn{4}{|l|}{ Apple } \\
\hline YYA12-1-1 & M. yunnanensis & Qujing, Yunnan & China \\
\hline YYA12-2-1a & M. yunnanensis & Qujing, Yunnan & China \\
\hline YYA13-2-1a & M. yunnanensis & Lijiang, Yunnan & China \\
\hline YYA13-3-1a & M. yunnanensis & Lijiang, Yunnan & China \\
\hline YYA13-4-1a & M. yunnanensis & Lijiang, Yunnan & China \\
\hline YYA13-5-1a & M. yunnanensis & Lijiang, Yunnan & China \\
\hline \multicolumn{4}{|l|}{ Pear } \\
\hline YYR12-1-2a & M. yunnanensis & Qujing, Yunnan & China \\
\hline YYR12-3-1b & M. yunnanensis & Qujing, Yunnan & China \\
\hline YYR12-7-1a & M. yunnanensis & Qujing, Yunnan & China \\
\hline YYR12-8-2a & M. yunnanensis & Qujing, Yunnan & China \\
\hline YYR12-9-5b & M. yunnanensis & Qujing, Yunnan & China \\
\hline YYR13-1-1a & M. yunnanensis & Lijiang, Yunnan & China \\
\hline \multicolumn{4}{|l|}{ Cherry } \\
\hline MCC13-7 & M. mumecola & Banan, Chongqing & China \\
\hline CCC14-5-2 & M. fructicola & Banan, Chongqing & China \\
\hline CCC14-7 & M. fructicola & Banan, Chongqing & China \\
\hline CCC14-11-1 & M. fructicola & Banan, Chongqing & China \\
\hline CCC14-13-1 & M. fructicola & Banan, Chongqing & China \\
\hline
\end{tabular}

${ }^{\mathrm{z}} \mathrm{NA}=$ not available. 
China Group Co., Ltd.). Mean values of conidia dimensions were determined based on values of 60 conidia from each isolate. To investigate conidia germination, conidia were spread onto PDA medium ( $\leq 2 \mathrm{~mm}$ in medium thickness) and incubated at $22^{\circ} \mathrm{C}$ in darkness. After $4 \mathrm{~h}$ of incubation, germ tubes were observed with a BK5000TR compound light microscope (Chongqing Optical \& Electrical Instrument Co., Ltd.).

Molecular identification of representative isolates with distinct morphological characteristics. Representative isolates of morphologically distinct phenotypes were also identified to species level by sequencing the ribosomal internal transcribed spacer (ITS)1-5.8SITS2 region and using a polymerase chain reaction (PCR) protocol described previously (Hu et al. 2011b), with minor modifications. In brief, single agar plugs containing actively growing mycelium were transferred on PDA medium covered with cellophane membrane and incubated for 3 days. Mycelia were collected from the surface of the cellophane membrane using a scalpel, and genomic DNA was subsequently extracted using the Easypure Plant Genomic DNA Extraction Kit (TransGen Biotech, Beijing) according to the manufacturer's instructions. For PCR-based identification, multiplex PCR was performed with primers PRCmon-F, PRCmon-R, Cola-F, Ensis-F, Mume-F, and Mon-R in a volume of $25 \mu l$ containing $1 \times$ PCR buffer, $20 \mathrm{ng}$ of DNA, $0.16 \mu \mathrm{M}$ each primer, $200 \mu \mathrm{M}$ each $\mathrm{dNTP}$, and $1 \mathrm{U}$ of Taq DNA polymerase (TransGen Biotech) (Hu et al. 2011b). The PCR reaction was carried out with the following parameters: an initial denaturation at $94^{\circ} \mathrm{C}$ for $3 \mathrm{~min} ; 30$ cycles of $30 \mathrm{~s}$ at $94^{\circ} \mathrm{C}, 30 \mathrm{~s}$ at $60^{\circ} \mathrm{C}$, and $2 \mathrm{~min}$ at $72^{\circ} \mathrm{C}$; and a final extension step at $72^{\circ} \mathrm{C}$ for $5 \mathrm{~min}$. The ITS1-5.8S-ITS2 region was amplified from genomic DNA with primer pair ITS1/ITS4, as described previously (White et al. 1990). All amplifications were performed in an iCycler thermal cycler (Bio-Rad Laboratories Inc., Hercules, CA). Amplification products were loaded onto $1.2 \%$ agarose gels (AGAROS G-10, GENE COMPANY, Hong Kong, China) immersed in $0.5 \times$ Tris-borate-EDTA buffer for $1 \mathrm{~h}$ at $100 \mathrm{v}$. The gels were then stained with ethidium bromide and photographed under UV light using an Alphalmager EP image acquisition system (Alpha Innotec, Santa Clara, CA). PCR products of ITS region were directly sent to Beijing Genomics Institute (Shenzhen, China) for sequencing.

Sequencing the glyceraldehyde-3-phosphate dehydrogenase and $\beta$-tubulin gene fragments. Evolutionary relationships of Monilinia spp. from plum and other hosts were investigated based on glyceraldehyde-3-phosphate dehydrogenase $(G 3 P D H)$ and $\beta$-tubulin (TUB2) gene fragments. In total, 47 Monilinia spp. isolates from six hosts (Table 1) were included. A G3PDH fragment (768 bp in size, approximately $70 \%$ of the whole $G 3 P D H$ gene) was amplified with primer pair Mon-G3pdhF/Mon-G3pdhR (Hu et al. 2011b). Similarly, a fragment of $T U B 2$ gene (1,630 bp in length, approximately $92 \%$ ) was amplified by using primer pair Mon-TubF1/Mon-TubR 1 (Hu et al. 2011b). PCR volumes were $50 \mu$ l, containing $1 \times$ PCR buffer, $50 \mathrm{ng}$ of DNA, $0.4 \mu \mathrm{M}$ each primer, $200 \mu \mathrm{M}$ each dNTP, and 2.5 U of Taq DNA polymerase (TransGen Biotech). All amplifications were performed in an iCycler thermal cycler (Bio-Rad Laboratories Inc.). The parameters for amplifying G3PDH and TUB2 were almost identical to the parameters used for ITS region amplification, except that the annealing temperature was decreased to $50^{\circ} \mathrm{C}$. PCR products were sent to the Beijing Genomics Institute for sequencing.

Construction of phylogenetic trees. Phylogenetic analysis was conducted for the combined data matrix of the two coding loci (G3PDH and TUB2). Multiple alignments were conducted using DNASTAR (DNASTAR Inc., Nevada, City CA) and CLUSTAL X 1.81(Jeanmougin et al. 1998; Thompson et al. 1997). The maximum parsimony (MP) tree was constructed by Mega 5.0 software (Tamura et al. 2011) using the MP method (Thompson et al. 1997) and computed with the Subtree-Prunning-Regrafting (level =1). A brand-swapping method with initial trees was generated by random addition (100 reps). A complete deletion option was used to treat gaps or missing data and the reliability of clusters was evaluated by bootstrapping with 1,000 replicates. The maximum likelihood (ML) tree was constructed by the ML method using PhyML 3.0 software (Guindon and Gascuel 2003), with a model that was determined by Find Best DNA/Protein Models program in Software Mega 5.0 (Tamura et al. 2011): TN93 + G substitution matrix, estimated $\mathrm{Ts} / \mathrm{Tv}$ ratio, fixed proportion of invariable sites $(\mathrm{p}$-invar $=0.00)$, and $\gamma$-distributed substitution rates $(\alpha=0.21)$. The reliability of internal branches was evaluated based on approximate likelihood ratio test (SH-like aLRT) statistics (Anisimova and Gascuel 2006).

\section{Results}

Morphology of Monilinia isolates from plum. Based on the colony morphology on PDA medium, the 112 Monilinia isolates from plum were divided into three phenotypes. Phenotype 1 colonies were
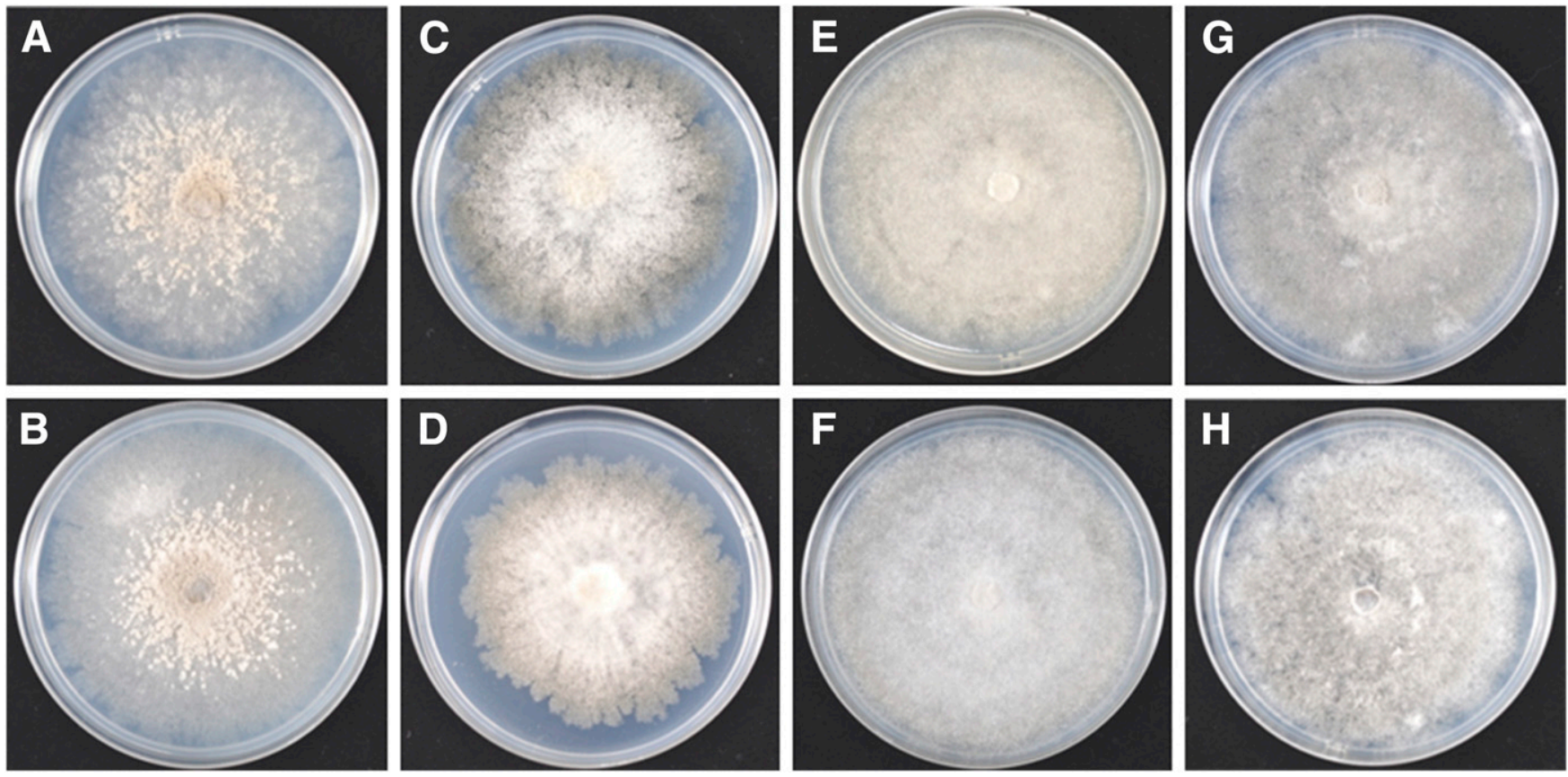

Fig. 1. Colony morphologies associated with phenotypes on potato dextrose agar medium. Isolates were grown for 5 days at $22^{\circ} \mathrm{C}$ in darkness. $\mathbf{A}$ and $\mathbf{B}$, Phenotype 1 isolates CCM13-3-1 and CCM13-5-1; C and D, phenotype 2 isolates MCM13-14-1 and MCM13-6-1; E and F, phenotype 3 isolates YYM12-57-3 and YYM12-57-4; and G and H, M. polystroma isolates CBS86 and CBS87, respectively. 
gray to brown, with apparent mycelium tufts of sporulation (Fig. 1A and B). Phenotype 2 colonies showed gray to brown, sometimes even green mycelia with lobbed margins and less sporulation compared with phenotype 1 colonies (Fig. 1C and D). Mycelia of phenotype 3 colonies were whitish to grayish, sometimes even greenish and lacked areas of sporulation (Fig. 1E and F). Two representative isolates from each phenotype (i.e., CCM13-3-1 and CCM13-5-1 representing phenotype 1, MCM13-6-1 and MCM13-14-1 representing phenotype 2, and YYM12-57-3 and YYM12-57-4 representing phenotype 3) were selected for further investigation. The representative isolates for each phenotype were selected from different locations whenever possible. Colonies of phenotype 1 and 2 isolates rarely produced stroma but colonies of phenotype 3 isolates produced ample stromata (Fig. 2A to F). Mycelial growth of phenotype 2 isolates was generally slower growing compared with isolates from phenotypes 1 and 3 and compared with $M$. polystroma reference isolates (Table 2). M. polystroma isolates grew fastest on PDA (Table 2).

Pathogenicity and appearance of isolates of phenotypes 1 to 3 on detached plum fruit. Brown rot symptoms were observed on inoculated plum fruit with all phenotypes, while the control fruit remained healthy. All isolates produced large numbers of gray to brown mycelia tufts with areas of sporulation on the inoculated plum fruit, though minor differences were observed (Fig. 3). Phenotype 1 and reference $M$. polystroma isolates produced large numbers of brown, loose sporodochia, which were more compact for M. polystroma isolates than for phenotype 1 isolates (Fig. 3A, B, $\mathrm{G}$, and $\mathrm{H}$ ). The sporodochia produced by isolates of phenotype 2 appeared gray to dark green instead of general brown, and the structure was less dense (Fig. 3C and D). In contrast, phenotype 3 isolates
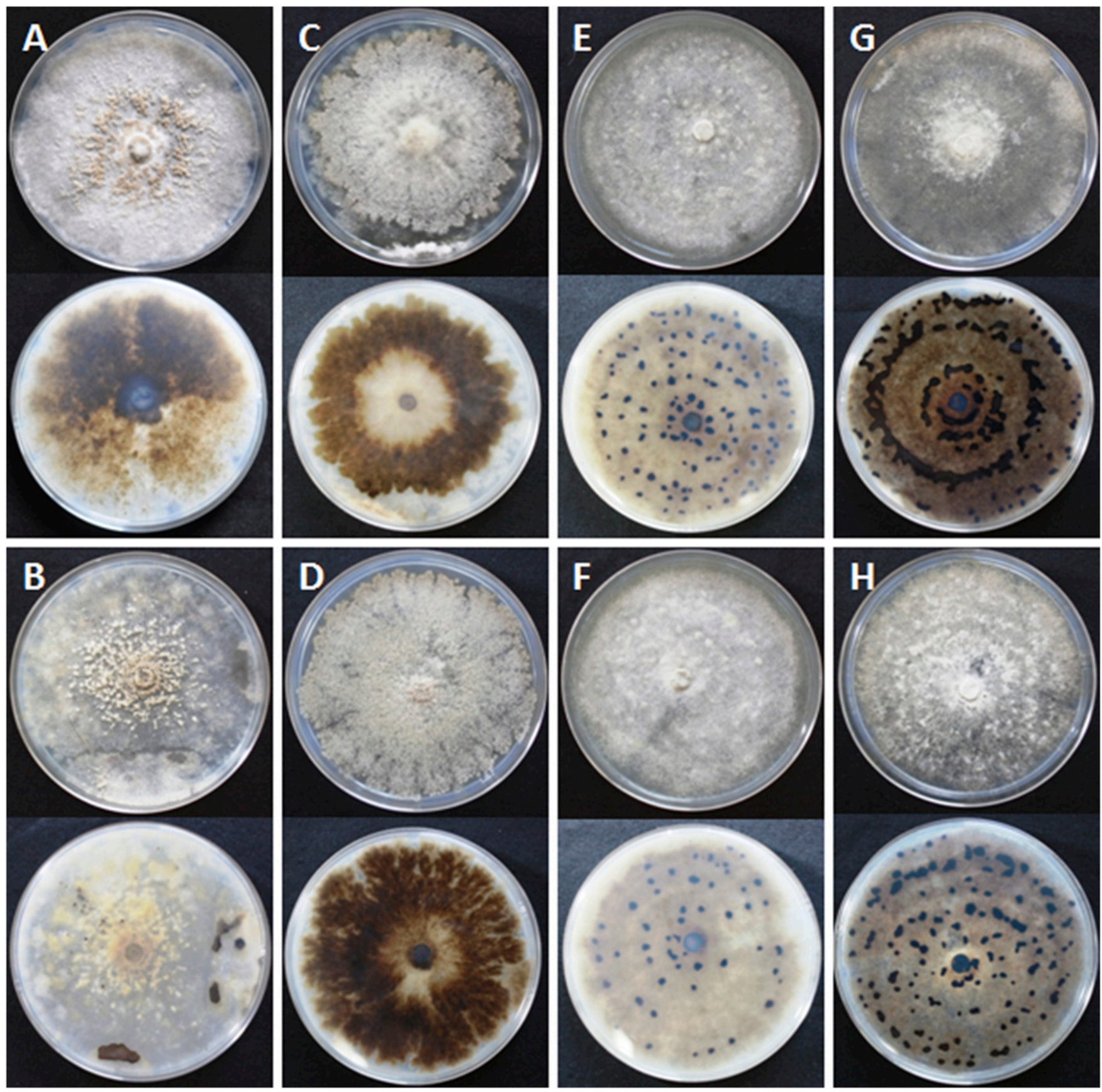

Fig. 2. Production of stromata of phenotypes 1 to 3 on potato dextrose agar medium. Colonies were incubated for 4 weeks at $22^{\circ} \mathrm{C}$ in darkness. A and B, Phenotype 1 isolates CCM13-3-1 and CCM13-5-1; C and D, phenotype 2 isolates MCM13-14-1 and MCM13-6-1; E and F, phenotype 3 isolates YYM12-57-3 and YYM12-57-4; and G and H, M. polystroma isolates CBS86 and CBS87, respectively. Front and back view of each plate is illustrated. 
produced fewer but larger sporodochia which were brownish in color and compact in shape (Fig. 3E and F).

Size, shape, and germination pattern of conidia collected from plum. Isolates of phenotypes 1 and 3 as well as isolates of M. polystroma produced similar cylindrical or lemon-shaped conidia but phenotype 2 isolates produced globose to subglobose conidia (Fig. 4). Conidia produced by phenotype 2 isolates were larger than those of other isolates (Fig. 4C and D; Table 2). The conidia size of phenotype 3 isolates was similar to that of $M$. polystroma. Conidia of phenotype 1 isolates were smallest among the tested isolates (Fig. 4A, B, E-H; Table 2).

The conidia of different phenotypes of isolates had different germination patterns (Fig. 5). Isolates of phenotype 1 produced one germ tube per conidium, which frequently initiated from the middle of the conidia (Fig. 5A and B). Type 3 isolates produced one or two germ tubes (Fig. 5E and F), while M. polystroma isolates generally produced two germ tubes per conidium (Fig. 5G and $\mathrm{H}$ ), and these germ tubes generally developed from the pointy sides of the conidia (Fig. 5E-H). Interestingly, isolates of phenotype 2 frequently produced more than two germ tubes per conidium (Fig. 5C and D).

Based on the above biological observations, the characteristics of isolates of phenotypes 1,2 , and 3 were consistent with that of M. fructicola, Monilia mumecola, and Monilia yunnanensis, which were described as the causal agents of peach brown rot in China previously (Hu et al. 2011b). The morphology-based species identification was subsequently confirmed by ribosomal ITS1-5.8S-ITS2 sequencing and a previously published, PCR-based method (Hu et al. 2011b).

Identification of representative isolates by species-specific PCR and ITS sequencing. A multiplex PCR method according to Hu et al. (2011b) was used to differentiate isolates of phenotypes 1, 2, and 3. Primer pair PCRmon-F/PCRmon-R amplified a 354-bp

Table 2. In vitro mycelia growth rate and size of plum fruit-derived conidia

\begin{tabular}{|c|c|c|c|c|c|}
\hline \multirow[b]{2}{*}{ Isolate } & \multirow[b]{2}{*}{ Colony morphology ${ }^{z}$} & \multicolumn{2}{|c|}{$\begin{array}{l}\text { In vitro colony growth rate } \\
\left(\mathbf{m m} \text { day }^{-1}\right)^{\mathbf{x}}\end{array}$} & \multicolumn{2}{|c|}{$\begin{array}{l}\text { Plum fruit-derived conidia } \\
\text { size }(\mu \mathrm{m})^{\mathrm{y}}\end{array}$} \\
\hline & & Mean \pm SD & Range & Mean $(\mathbf{W} \times \mathbf{L})$ & Range \\
\hline$\overline{\mathrm{CCM} 13-3-1}$ & $\begin{array}{l}\text { Gray to brown with apparent mycelium tufts } \\
\text { of sporulation }\end{array}$ & $13.2 \pm 1.8 \mathrm{bcd}$ & $11.2-15.0$ & $9.9 \times 13.9$ & $6.3-14.3 \times 8.0-21.3$ \\
\hline CCM13-5-1 & $\ldots$ & $15.0 \pm 0.7 \mathrm{abc}$ & $14.3-16.0$ & $10.2 \times 13.5$ & $5.3-13.0 \times 8.3-18.3$ \\
\hline MCM13-6-1 & $\begin{array}{l}\text { Gray to brown, or even green mycelium with } \\
\text { lobbed margins and less sporulation }\end{array}$ & $12.3 \pm 2.5 \mathrm{~d}$ & $8.6-14.3$ & $16.4 \times 20.1$ & $10.8-25.0 \times 12.5-25.0$ \\
\hline MCM13-14-1 & $\ldots$ & $12.7 \pm 2.6 \mathrm{~cd}$ & $10.7-16.5$ & $14.5 \times 20.9$ & $10.3-19.0 \times 12.8-31.5$ \\
\hline YYM12-57-3 & $\begin{array}{l}\text { Whitish to grayish, or even greenish and } \\
\text { lacked areas of sporulation }\end{array}$ & $15.2 \pm 0.4 \mathrm{ab}$ & $14.8-15.7$ & $10.9 \times 17.3$ & $7.8-15.0 \times 13.8-21.8$ \\
\hline YYM12-57-4 & $\ldots$ & $14.8 \pm 1.1 \mathrm{abc}$ & $13.7-16.3$ & $9.9 \times 16.0$ & $7.5-13.0 \times 12.5-20.0$ \\
\hline CBS86 & $\begin{array}{l}\text { Similar to that of YYM12-57-3 and } \\
\text { YYM12-57-4 }\end{array}$ & $15.5 \pm 0.6 \mathrm{a}$ & $14.7-16.2$ & $11.2 \times 17.0$ & $7.8-17.5 \times 11.2-23.8$ \\
\hline CBS87 & $\ldots$ & $15.9 \pm 1.2 \mathrm{a}$ & $14.8-17.5$ & $10.5 \times 16.3$ & $7.5-12.8 \times 10.0-22.5$ \\
\hline
\end{tabular}

${ }^{\mathrm{x}}$ Isolates were grown on potato dextrose agar (PDA) at $22^{\circ} \mathrm{C}$ in the dark, the diameter of colonies was measured at the second, third, fourth, and fifth day after inoculation, growth rate was calculated as the mean values of daily growth values, and three replications were performed for each isolate. Mean $\pm \mathrm{SD}=$ mean value with standard deviation; values within the same column followed by the same letters are not significantly different based on the analysis of least significant difference test at $P=0.05$ in SPSS.

y Conidia were harvested from inoculated plum fruit, and the conidia sizes $(\mathrm{W}=$ width and $\mathrm{L}=$ length) were determined based on at least 60 conidia from each isolates.

z Colony morphology on PDA.
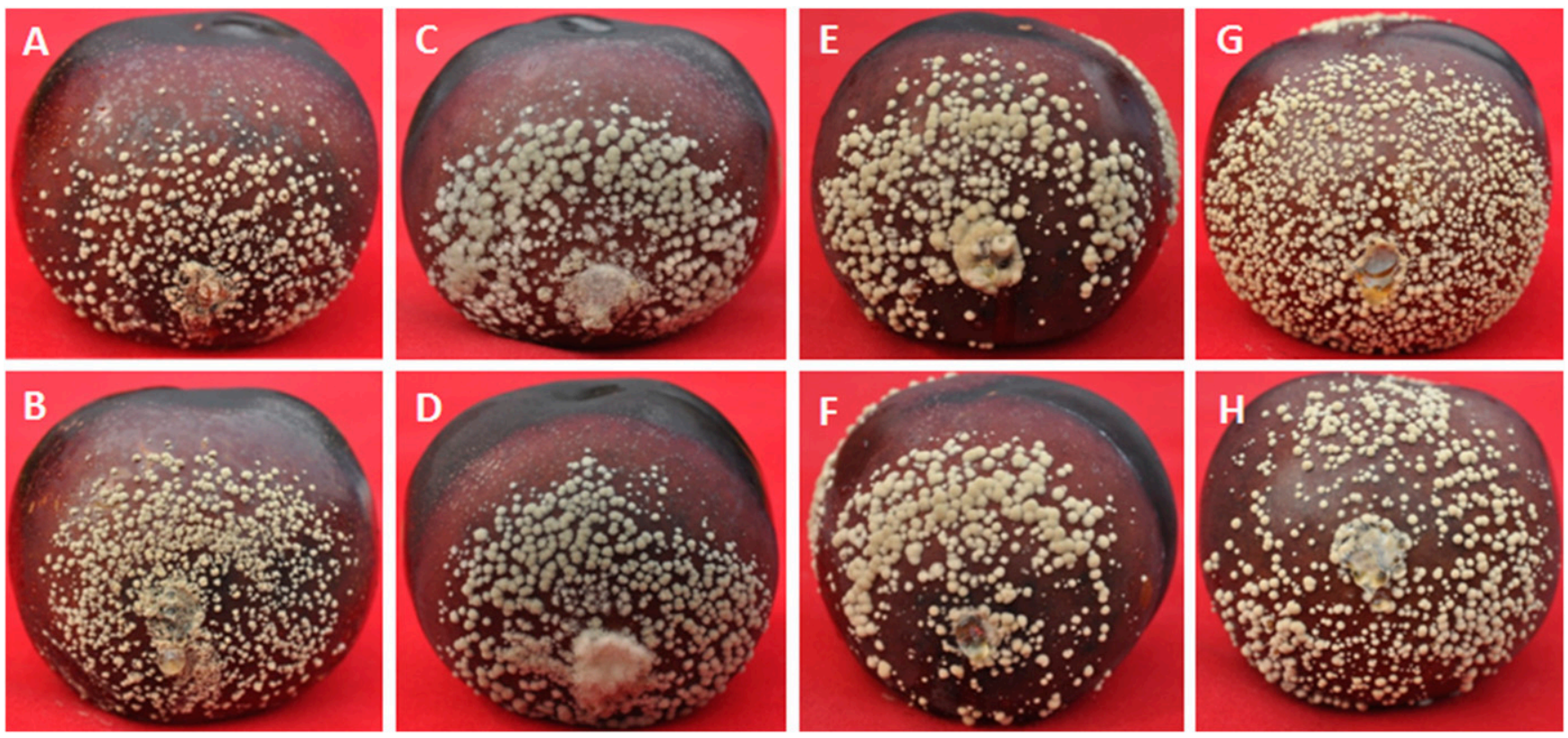

Fig. 3. Symptoms of four Monilinia spp. on plum fruit (Heibuling). Inoculated fruit was incubated at $22^{\circ} \mathrm{C}$ for 4 days under $12 \mathrm{~h}$ of light and $12 \mathrm{~h}$ of darkness. A and B, Phenotype 1 isolates CCM13-3-1 and CCM13-5-1; C and D, phenotype 2 isolates MCM13-14-1 and MCM13-6-1; E and F, phenotype 3 isolates YYM12-57-3 and YYM12-57-4; and G and H, M. polystroma isolate CBS86 and CBS87, respectively. 
fragment from the $G 3 P D H$ gene sequence from all phenotype 1, 2, 3 , and M. polystroma reference isolates, indicating that all of them were Monilinia spp. Reverse primer Mon-R and species-specific forward primers Cola-F, Mume-F, and Ensis-F amplified fragments of 534, 712, or 237 bp in length (Fig. 6) for phenotypes 1, 2, and 3, identifying them as M. fructicola, M. mumecola, and M. yunnanensis, respectively. The PCR method was developed previously to distinguish Chinese peach brown rot fungi and did not amplify a species-specific fragment from $M$. polystroma isolates (Fig. 6).

ITS1-5.8S-ITS2 sequences also identified phenotypes 1, 2, and 3 as $M$. fructicola, M. mumecola, and $M$. yunnanensis, respectively. The ITS sequences of two phenotype 1 isolates were identical and showed $100 \%$ identity with that of M. fructicola isolates from the United States (accession number KJ5541032), Serbia (KC544809 and JN176564), China (HQ893747, HQ893748, FJ515894, and AM887528), Slovenia (GU967379), Italy (FJ411109), and Spain (EF207419) in BLAST searches. The two isolates of phenotype 2 displayed one nucleotide difference in their ITS sequences. Both genotypes were described before in $\mathrm{M}$. mumecola isolates $(\mathrm{Hu}$ et al. 2011b); that is, isolates MCM13-14-1 and MCM13-6-1 had a cytosine (C) and thymine (T) at the 442 position which were exactly the same as ITS sequences of $M$. mumecola isolates ML-1a (HQ908786) and HXL10-1a (HQ908787), respectively. The two isolates of phenotype 3 collected from Yunnan province had identical ITS sequences and showed the highest similarity (100\%)
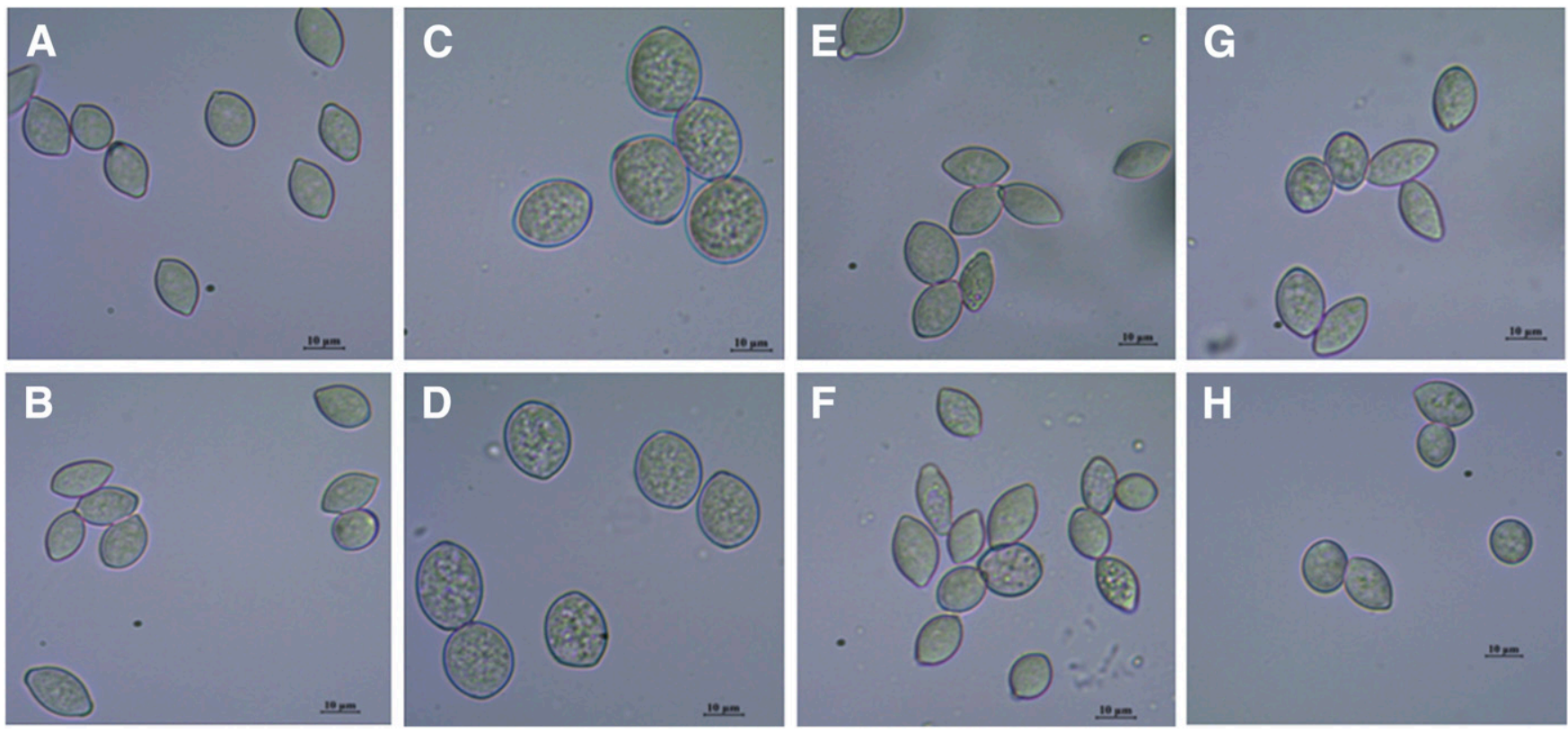

Fig. 4. Conidia morphology characters of the four Monilinia spp. from plum on potato dextrose agar. Photos were taken under $\times 400$ microscope; bars represent $10 \mu \mathrm{m}$. A and B, Phenotype 1 isolates CCM13-3-1 and CCM13-5-1; C and D, phenotype 2 isolates MCM13-14-1 and MCM13-6-1; E and F, phenotype 3 isolates YYM12-57-3 and YYM12-57-4; and $\mathbf{G}$ and $\mathbf{H}$, M. polystroma isolates CBS86 and CBS87, respectively.
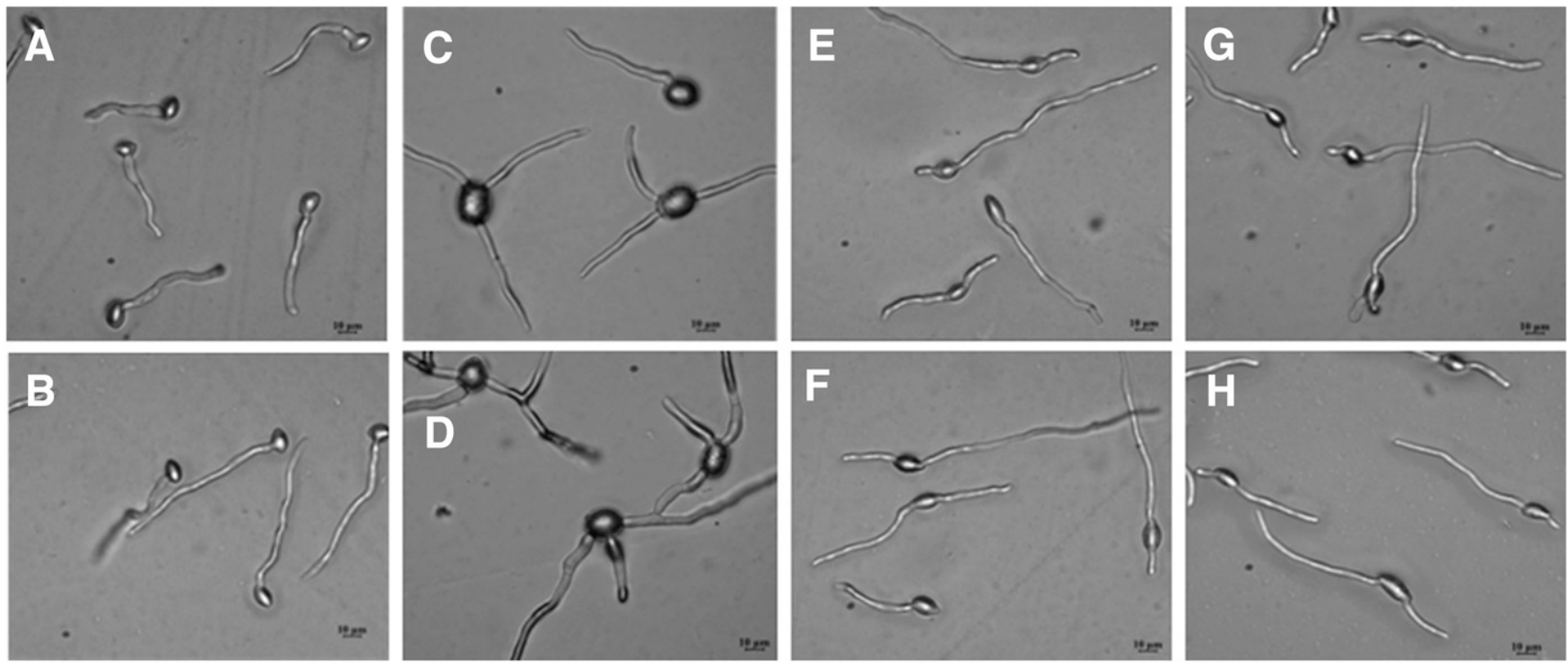

Fig. 5. Germ tube morphology of the four Monilinia spp. from plum. Conidia were incubated on potato dextrose agar at $22^{\circ} \mathrm{C}$ in darkness for $5 \mathrm{~h}$, conidia photos were taken under a $\times 200$ microscope, and bars represent $10 \mu \mathrm{m}$. A and B, Phenotype 1 isolates CCM13-3-1 and CCM13-5-1; C and D, phenotype 2 isolates MCM13-14-1 and MCM13-6-1; E and F, phenotype 3 isolates YYM12-57-3 and YYM12-57-4; and $\mathbf{G}$ and $\mathbf{H}$, M. polystroma isolates CBS86 and CBS8, respectively. 
with ITS sequence of $M$. yunnanensis isolate KY-1 from China (HQ908788). The two reference isolates of $M$. polystroma had the same ITS sequence compared with $M$. polystroma isolates from China (GU067539 and HQ856916), Serbia (JX315717), and Switzerland (JN128835).

Phylogenetic analysis of Monilinia spp. Phylogenetic analysis was conducted based on G3PDH and TUB2 sequences to illustrate the genetic and evolutionary relationships of isolates from plum and other hosts. Phylogenetic trees were constructed with the MP and ML methods and the topologies of the phylogenetic trees with two methods were concordant with each other, indicating that the evolutionary relationship of tested isolates was statistically supported (Fig. 7A and B). The topology of phylogenetic analysis revealed that the relationship between isolates was determined by species rather than their hosts or origins (Fig. 7). M. fructicola was the most basal clade; other clades of species were more derived but shared a single common ancestor with $M$. fructicola. The most derived clade was that of M. polystroma and Monilinia fructigena, which was phylogenetically closely related to $M$. yunnanensis. The clades containing Monilinia laxa and M. mumecola isolates were located between the M. fructicola and M. mumecola clades (Fig. 7). Within a species, there were some noteworthy variations. M. fructicola isolate Dmap3-08 from the United States, originating from peach, was genetically unique; but U.S. isolate Bmpc5, also from peach, was genetically very similar to Chinese isolate CCC14-11-1 from Cherry. M. mumecola isolate MCM13-9-1 collected from plum from Youyang, Chongqing municipality was genetically unique compared with other $M$. mumecola isolates. $M$. yunnanensis isolates were all from Yunnan province and genetically very similar to each other.

The phylogenetic analysis clustered isolates according to species rather than geographic or host origin. For example, M. fructicola isolate ZM09-2a from Zhejiang province clustered together with isolates from Chongqing municipality. Furthermore, isolate Bmpc5 from the United States grouped tightly with Chinese isolate CCC14-11-1 (Fig. 7). M. mumecola isolates MCM13-6-1 and MCC13-7 from Chongqing municipality grouped with five isolates from Hubei province (Fig. 7), and M. yunnanensis isolate YKG1061a from Kunming city (central Yunnan) was closely related to isolates from Lijiang city (Northwestern Yunnan) and Qujing city (Eastern Yunnan) (Fig. 7). In contrast, some isolates from different hosts shared very similar genetic backgrounds. For examples, M. fructicola isolate CCC14-5-2 from cherry grouped tightly with four other plum isolates, whereas the peach isolate ZM09-2a and the cherry isolate CCC14-13-1 clustered together with three other plum isolates (M. fructicola subgroup; Fig. 7). For M. mumecola, some isolates from apricot, peach, plum, and cherry clustered together and, for M. yunnanensis, many isolates from different hosts clustered together; for example, YYA13-4-1a, YYA13-2-1a, YYA12-1-1 (apple), and YKG10-61a (peach); YYM12-57-3 (plum) and YYR121-2a and YYR12-3-1b (pear) (M. yunnanensis subgroup; Fig. 7). These results indicate a lack of host or location specificity of isolates of the Monilinia spp. examined.

\section{Discussion}

Based on cultural characteristics and molecular analysis, three Monilinia spp. (i.e., M. fructicola, M. mumecola, and M. yunnanensis) were identified to cause brown rot diseases on plum in China. This information is of scientific and practical significance. Monilinia spp. may vary in their biology, epidemiology, and etiology, which are important considerations for disease management. For example, $M$. laxa is commonly found to infect flowers rather than fruit, while $M$. fructigena primarily causes fruit rot and, less frequently, flower infections. M. fructicola is almost exclusively found on fruit (Batra 1991). M. fructicola, M. laxa, and $M$. mumecola primarily infect stone fruit but $M$. fructigena and M. yunnanensis prefer pome fruit (Batra 1991; Hu et al. 2011b; Shao 2009; Zhao et al. 2013). The fact that plum in China is infected by three species may complicate disease management design. More information is necessary to determine species-specific host tissue preference, optimal weather conditions for infection, and the potential differences in fungicide sensitivity among species in order to design effective, integrated management strategies for brown rot disease on plum.

In addition to plum, Monilinia spp. have already been described causing brown rot diseases on many other fruit in China. M. fructicola was first reported in 2005 in Beijing, causing serious damage on peach and nectarine (Zhu et al. 2005). After that, $M$. fructicola was identified as the species that caused brown rot of peach in eastern ( $\mathrm{Li}$ and Chen 2009), southeastern (Hu et al. 2011a), central, and western China (Yin et al. 2013) and was identified as the most prevalent species causing brown rot disease on peach and nectarine in China (Hu et al. 2011b). M. fructicola was also reported to cause brown rot on Chinese dwarf cherry (Cerasus humilis) in northeastern China, where more than $80 \%$ fruit rot was observed (Xu et al. 2011). In this study, representative phenotype 1 isolates were identified as $M$. fructicola, and this species was formally verified as a new Monilinia sp. on plum in China.

M. mumecola was first isolated from P. mume in Japan in 1982 (Nakao 1992) but not identified to the species level until 2004 (Harada et al. 2004). Previous studies showed that M. mumecola can cause brown rot on stone fruit such as peach, nectarine $(\mathrm{Hu}$ et al. 2011b), apricot (P. armeniaca) (Yin et al. 2014b), and Chinese sour cherry (P. pseudocerasus) (Yin et al. 2014a). It also was described as the causal agent of brown rot on flowering quince (Chaenomeles lagenaria; Shao 2009).

M. mumecola typically produces larger conidia that can generate two or more germ tubes from individual conidia (Hu et al. 2011b; Yin et al. 2014a,b). In this study, phenotype 2 isolates produced the largest conidia, typically with two or more germ tubes. Molecular identification based on a multiplex PCR method and ITS sequencing also verified that phenotype 2 isolates were M. mumecola. To our knowledge, this is the first report of $M$. mumecola causing plum brown rot in China.

M. yunnanensis was first described as a causal agent of peach brown rot in 2011 (Hu et al. 2011b). Its genetically close relationship with $M$. polystroma and $M$. fructigena was reported previously ( $\mathrm{Hu}$ et al. 2011b) and was confirmed in the present study. The phenotype 3 isolates of this study were identified as $M$. yunnanensis based on biological and molecular characteristics. In 2013, M. yunnanensis

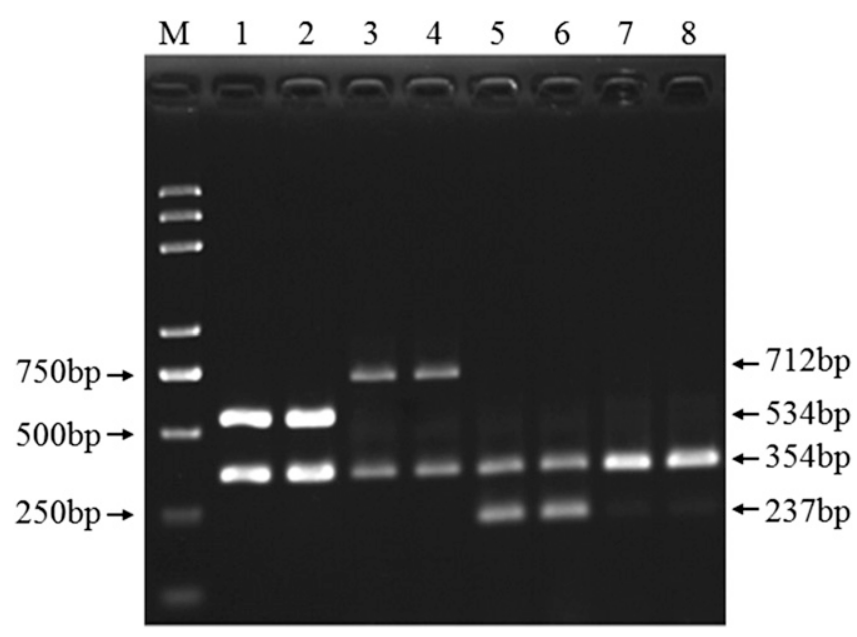

Fig. 6. Multiplex polymerase chain reaction (PCR) results for Monilinia spp. isolates from plum. Lanes 1 to 8: isolates CCM13-3-1, CCM13-5-1, MCM13-14-1, MCM136-1, YYM12-57-3, YYM12-57-4, CBS86, and CBS87. Trans 2K (TransGen Biotech, Beijing, China) molecular maker is shown at left. Primer pair PRCmon-F/PRCmon-R was used to produce a 354-bp fragment (lanes 1 to 8) from Monilinia spp. Reverse primer Mon-R and species-specific forward primers Cola-F, Mume-F, and Ensis-F produced amplicons 534 bp (lanes 1 and 2), 712 bp (lane 3 and 4), or 237 bp (lane 5 and 6 ) in length from phenotype 1, 2, or 3 isolates, respectively. No specific fragment was amplified from reference M. polystroma isolates (lanes 7 and 8). 
was reported to cause brown rot on Chinese hawthorn, Crataegus pinnatifida var. major (Zhao et al. 2013), in northern China. However, prior to the current study, this species had not been reported on plum. Morphologically, M. yunnanensis was very similar to M. polystroma: both species produced a large number of stromata on PDA, the mycelia were gray to green, and generally aerial mycelia were undeveloped. Conidia of both species were similar in size, shape, and germination pattern. The PCR-based method did not distinguish $M$. polystroma from other species because the latter species produced amplification patterns not unique to this species. Distinction was only possible based on ITS1-5.8S-ITS2 sequences.

Our phylogenetic analysis of isolates from plum revealed genetic relationships between species that were similar to previous findings describing isolates from peach (Hu et al. 2011b). M. fructicola was genetically most distant from other Monilinia spp., while $M$. yunnanensis was closely related to $M$. polystroma and $M$. fructigena. Isolates of $M$. mumecola and M. laxa clustered between the extremes. The clustering of isolates was species specific rather than host or location specific. Single clades were made of M. polystroma and M. fructigena isolates in both MP and ML trees. This is consistent with the observation that these two species were the most closely related species in Monilinia spp. (Hu et al. 2011b; van Leeuwen et al. 2002). Monilinia spp. occur in preferred geographical regions. For example, M. fructicola is widely found in North and South
America, Europe, Asia, and Australia; M. fructigena is frequently found in temperature regions of Europe, eastern and southern Asia, northern Africa and South America; and M. laxa is usually found in Asia, the western Americas, Australia, and New Zealand. M. mumecola and M. polystroma are found in east Asia (China and Japan) and M. yunnanensis has only been found in China. A preference in origin of Monilinia spp. of plum was also observed in this study; for example, $M$. fructicola was detected in all four provinces and the municipality included in this study; M. mumecola in Hubei province and Chongqing municipality; and M. yunnanensis only in Yunnan province. This geographical distribution may be related to the chronological origination of individual species in the evolutionary history of Monilinia spp. It is also possible that fitness differences were responsible for the observed distribution. Nevertheless, isolates of the same species but from different geographical areas or hosts clustered together, suggesting that host or geographical environments did not play a significant role for the evolution of Monilinia spp.

In the present study, samples were collected from three provinces and one municipality. These areas reflect only 4 of 20 major plumproducing regions in China. Species other than $M$. fructicola, M. mumecola, M. yunnanensis, and M. polystroma may exist in China, and further sampling in other areas will need to be carried out in the future. It is becoming more evident, though, that M. polystroma is a rather rare Monilinia sp. in China.

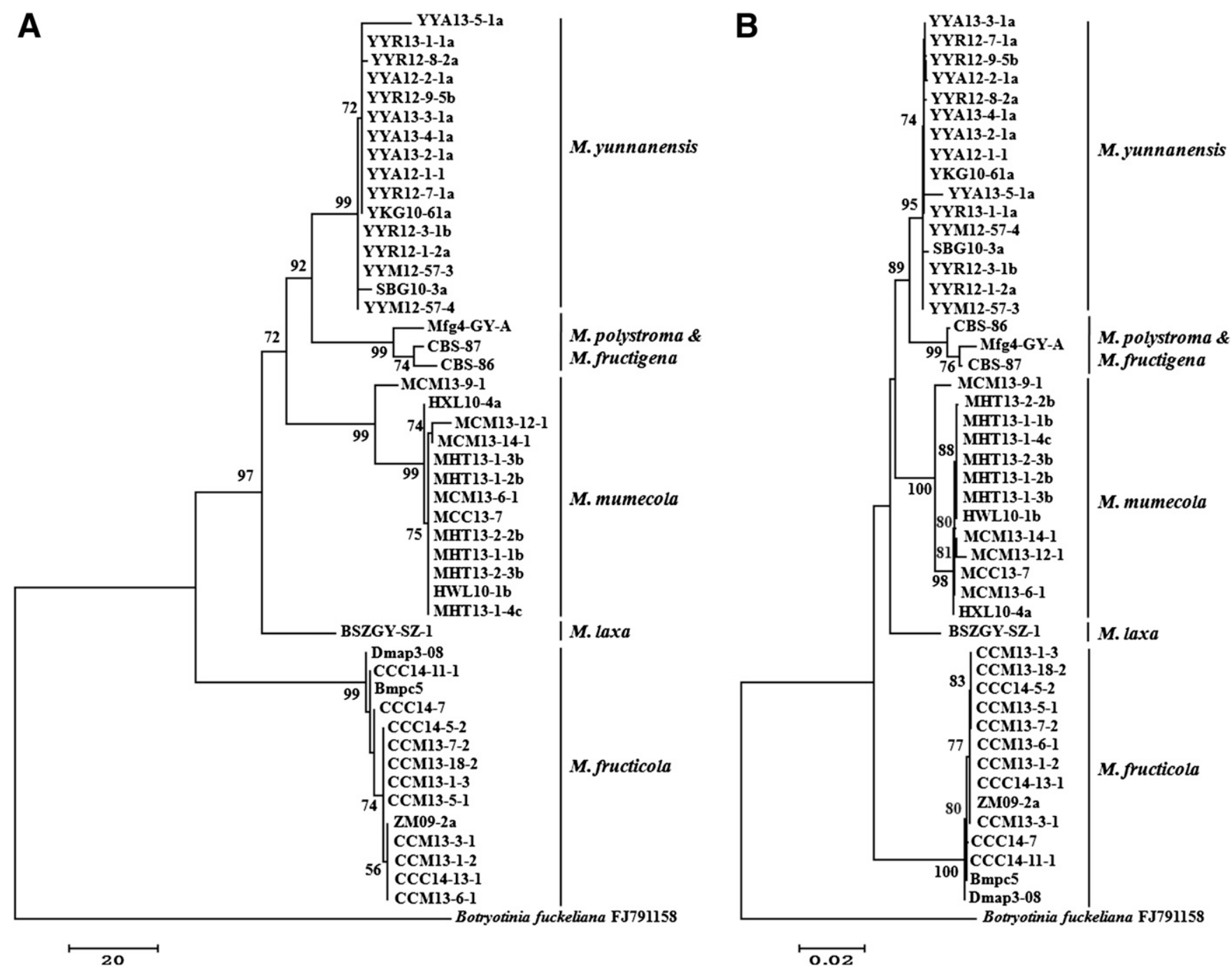

Fig. 7. Phylogenetic trees of 47 Monilinia spp. isolates and one outgroup species (Botryotinia fuckeliana). A, Maximum parsimony and B, maximum likelihood trees inferred from the data set containing the combined DNA sequences of glyceraldehyde-3-phosphate dehydrogenase (G3PDH) and $\beta$-tubulin (TUB2), with 2,208 characters, 198 of with were informative. Numbers labeled at each node indicate bootstrap values (\%) from 1,000 replicates. 


\section{Acknowledgments}

This material is based upon work supported by the Project of National Natural Science Foundation of China (numbers 31401686 and 31371896).

\section{Literature Cited}

Anisimova, M., and Gascuel, O. 2006. Approximate likelihood-ratio test for branches: A fast, accurate, and powerful alternative. Syst. Biol. 55:539-552.

Batra, L. R. 1991. World Species of Monilinia (Fungi): Their Ecology, Biosystematics and Control. Mycologia Memoir 16. J. Cramer, Berlin, New York.

Guindon, S., and Gascuel, O. 2003. A simple, fast, and accurate algorithm to estimate large phylogenies by maximum likelihood. Syst. Biol. 52:696-704.

Harada, Y., Nakao, S., Sasaki, M., Sasaki, Y., and Ichihashi, Y. a. 2004. Monilia mumecola, a new brown rot fungus on Prunus mume in Japan. J. Gen. Plant Pathol. 70:297-307.

Hu, M.-J., Chen, S. N., Liu, X. L., Yin, L. F., and Luo, C. X. 2011a. First report of brown rot of peach caused by Monilinia fructicola in southeastern China. Plant Dis. 95:225.

Hu, M. J., Cox, K. D., Schnabel, G., and Luo, C. X. 2011b. Monilinia species causing brown rot of peach in China. PLoS One 6:e24990.

Jeanmougin, F., Thompson, J. D., Gouy, M., Higgins, D. G., and Gibson, T. J. 1998. Multiple sequence alignment with Clustal X. Trends Biochem. Sci. 23: 403-405.

Li, S., and Chen, C. 2009. Incidence and management of the peach fruit brown rot. Plant Prot. 35:134-139.

Nakao, S. 1992. Brown rot disease of Prunus mume caused by an undescribed Monilia fungus. Kongetsu-no-noyaku 1:92-95.

Shao, W. 2009. Etiology, occurrence and control of Papaya (Chaenomeles lagenaria) brown rot. Master thesis, Department of Plant Protection, Huazhong Agricultural University, Wuhan, China.

Tamura, K., Peterson, D., Peterson, N., Stecher, G., Nei, M., and Kumar, S. 2011. MEGA5: Molecular evolutionary genetics analysis using maximum likelihood, evolutionary distance, and maximum parsimony methods. Mol. Biol. Evol. 28: 2731-2739.
Thompson, J. D., Gibson, T. J., Plewniak, F., Jeanmougin, F., and Higgins, D. G. 1997. The CLUSTAL_X windows interface: Flexible strategies for multiple sequence alignment aided by quality analysis tools. Nucleic Acids Res. 25: 4876-4882.

Tian, L. H., Zhou, G. L., Huang, J. K., Qiu, S. H., Zhou, E. X., and Yi, J. P. 2008. Identification of Monilinia laxa from imported Chilean plum. Plant Quarantine 22:201-204.

van Leeuwen, G. C. M., Baayen, R. P., Holb, I. J., and Jeger, M. J. 2002. Distinction of the Asiatic brown rot fungus Monilia polystroma sp. nov. from M. fructigena. Mycol. Res. 106:444-451.

White, T. J., Bruns, T., Lee, S., and Taylor, J. 1990. Amplification and direct sequencing of fungal ribosomal RNA genes for phylogenetics. Pages 315-322 in: PCR Protocols: A Guide to Methods and Applications, Academic Press, San Diego.

Xiang, W. N. 1957. Reference of Mycology and Plant Pathology in China. Science Press, Beijing.

Xu, C., Zhou, Z., Wu, Y., Chi, F., and Zhang, H. 2011. Identification of the pathogen causing brown rot of Chinese Dwarf Cherry (Cerasus humilis). Acta Phytopathol. Sin. 41:626-630.

Yin, L. F., Chen, G. K., Chen, S. N., Du, S. F., Li, G. Q., and Luo, C. X. 2014a First report of brown rot caused by Monilia mumecola on Chinese sour cherry in Chongqing municipality, China. Plant Dis. 98:1009.

Yin, L. F., Chen, S. N., Cai, M. L., Li, G. Q., and Luo, C. X. 2014b. First report of brown rot of apricot caused by Monilia mumecola. Plant Dis. 98:694.

Yin, L. F., Chen, S. N., Yuan, N. N., Zhai, L. X., Li, G. Q., and Luo, C. X. 2013. First report of peach brown rot caused by Monilinia fructicola in central and western China. Plant Dis. 97:1255.

Zhang, H. L., Zhang, J. C., Gu, J. F., and Xu, Y. 2008. Isolation and identification of plum brown rot pathogen. China Fruits 2:68-69.

Zhao, Y. Z., Wang, D., and Liu, Z. H. 2013. First report of brown rot on Crataegus pinnatifida var. major caused by Monilia yunnanensis in China. Plant Dis. 97: 1249.

Zhu, X. Q., Chen, X. Y., Luo, Y., and Guo, L. Y. 2005. First report of Monilinia fructicola on peach and nectarine in China. Plant Pathol. 54:575.

Zhu, X. Q., and Guo, L. Y. 2010. First report of brown rot on plum caused by Monilia polystroma in China. Plant Dis. 94:478. 